\title{
Potencial undimotriz de la región de Tarapacá (Chile) como foco de fomento productivo
}

\section{Region of Tarapacá's wave energy potential as a focus for productive development}

\author{
Raúl Castillo Villagra ${ }^{1 *} \quad$ Vivian Segovia Barros ${ }^{1}$ \\ Gabriel Díaz Flores ${ }^{1} \quad$ Claudia Araya Medina ${ }^{1}$
}

Recibido 28 de enero de 2016, aceptado 6 de marzo de 2017

Received: January 28, 2016 Accepted: March 6, 2017

\section{RESUMEN}

Las energías marinas están siendo cada vez más consideradas en el mundo como un recurso importante y prometedor. Unas de ellas, la energía undimotriz, utiliza la energía mecánica proveniente del movimiento de las olas del mar para la generación de electricidad. Este artículo presenta una revisión bibliográfica de las siguientes temáticas: situación energética mundial, beneficios en la recolección de energía undimotriz y tipos de convertidores utilizados actualmente. Chile presenta una situación energética que requiere el uso de nuevas tecnologías. La región de Tarapacá posee un potencial considerable de generación de electricidad por medio de las olas, por lo que el aprovechamiento de este tipo de energía se presenta como una opción altamente vinculante con las industrias que forman parte del motor económico de la región.

Palabras clave: Energía, ERNC, energía undimotriz, potencial undimotriz, convertidor undimotriz, potencial eléctrico, fomento productivo, región de Tarapacá, Chile.

\begin{abstract}
Marine energies are being increasingly considered worldwide as a major and promising resource. One of them, wave energy, captures the mechanical energy of waves to generate electricity. This paper presents a review of the following topics: global energy situation, advantages and currently used types of wave energy converters. Chile possesses an energy situation that requires the use of new technologies. The region of Tarapacá has a considerable potential for generating electricity from waves, so using this type of energy is presented as a highly relatable option with industries that are part of the major economic driver of the region.
\end{abstract}

Keywords: Energy, NCRE, wave energy, wave power, wave energy converter, electric potential, productive development, region of Tarapacá, Chile.

\section{INTRODUCCIÓN}

Disponer de energía se considera una condición fundamental para el crecimiento, desarrollo económico y avances sociales [1]. El sector energético es fundamental en la vida de las personas, considerando la energía como fuente o recurso necesario para la alimentación, transporte y funcionamiento productivo [2].

Durante décadas se consideró a los hidrocarburos como una fuente energética abundante y barata, que

\footnotetext{
1 Facultad de ingeniería y arquitectura. Universidad Arturo Prat. Av. Arturo Prat 2120. Iquique, Chile. E-mail: raucasti@unap.cl; vsegovia@unap.cl; diazfloresgabriel@gmail.com; claudia.araya.medina.92@gmail.com

* Autor de correspondencia
} 
daba respuesta a las necesidades del crecimiento global. Sin embargo, el crecimiento del consumo energético de algunos países y la progresiva urbanización mundial han creado un mercado de combustibles mucho más volátil y altos precios de la energía [2].

El reto que enfrenta Chile es el desarrollo de energía confiable, sustentable, equilibrada y que garantice al país mayor soberanía en sus necesidades energéticas. Según el Balance nacional de energía (BNE, 2012), en Chile se importa cerca del 60\% de la energía primaria, por lo que hace al mercado energético chileno susceptible a la volatilidad de los mercados internacionales, sin considerar las restricciones de abastecimiento, por fenómenos políticos, climáticos o económicos [2].

El incremento en el precio de la energía eléctrica es un tema relevante a nivel país, entre los años 2006 y 2013, el suministro eléctrico para familias, comercio y pequeñas empresas aumentó cerca de US\$ $63 \mathrm{MWh}$ y se estima una evolución de 34\% para los próximos 10 años. Además, el crecimiento sostenido en el consumo eléctrico proyecta una demanda cercana a 100 TWh para el año 2020 [2], y a nivel mundial el año 2040 será $30 \%$ mayor que el 2010 [1].

Las metas planteadas en la Hoja de Ruta 2050 proponen que para el año 2050, 70\% de la energía inyectada a los sistemas interconectados provenga de Energía Renovable No Convencional (ERNC). La política principal se ha centrado en energía solar y eólica, complementado con recurso fuentes hidroeléctricas [3].

Para alcanzar los porcentajes deseados, es necesario explotar otras formas de energía menos desarrolladas en el presente pero con alto potencial [4-6], como lo son las energías marinas contenidas en las olas, mareas, gradiente salinidad, y diferenciales de temperatura en el océano [7]. Entre estas diversas energías, la de tipo undimotriz es una fuente energética 'poco utilizada, a pesar del considerable potencial que presenta a nivel mundial. Por ende, a continuación, se exponen las bases teóricas de la energía undimotriz a través de una recopilación de información proveniente de documentos científicos y estrategias gubernamentales, que permita el posterior análisis del impacto que generaría su potencial aprovechamiento.

\section{ANÁLISIS TEÓRICO/DESARROLLO}

Se tiene la percepción de que la energía undimotriz es un campo reciente de investigación energética, sin embargo, la primera patente de explotación de energía de las olas data del año 1799 en Francia [8]. En efecto, la investigación moderna de la producción de electricidad por medio de las olas fue impulsada debido a la crisis del petróleo de la década de 1970 [9]. A pesar de mantenerse en una etapa aún exploratoria, presenta amplias posibilidades para el futuro gracias a su enorme potencial en la producción eléctrica [10-20]. De hecho, el recurso undimotriz global está estimado en 3702 GW de potencia bruta y $2985 \mathrm{GW}$ potencial útil, excluyendo olas con energía inferior a $5 \mathrm{~kW} / \mathrm{m}$ y posibles áreas cubiertas de hielo [21]. Las zonas con mayores potencias medias en las olas en latitudes medias, entre $\operatorname{los} 30^{\circ}$ y $60^{\circ}$ de latitud, como se muestra en la Figura 1.

Se estima que el potencial de recurso undimotriz en la costa oeste de América del Sur es de 325 GW de potencia bruta [21], y Chile posee $240 \mathrm{GW}$ [22] del potencial energético de este.

En la última década, la atención global se ha centrado en el cambio climático y el incremento de los niveles de $\mathrm{CO}_{2}$, enfocándose en la generación de electricidad con recursos renovables, siendo nuevamente un área importante de investigación.

\section{Beneficios}

Utilizar las olas como un recurso energético renovable ofrece importantes ventajas en los métodos de generación de energía, donde se incluyen los siguientes:

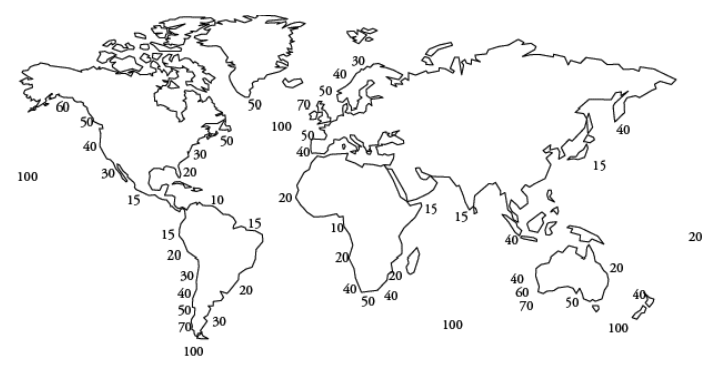

Figura 1. Distribución de energía undimotriz en mar abierto, potencia de olas expresado en $\mathrm{kW} / \mathrm{m}$ de longitud de cresta de ola [8]. 
- Las olas del mar ofrecen abundante energía y de alta densidad, siendo un recurso predecible conociendo las variables atmosféricas y climáticas [23-26].

- El leve impacto ambiental negativo en comparación con otras fuentes de energías convencionales [23-27].

- Variabilidad estacional natural de la energía undimotriz, que presenta una tendencia similar a la demanda de electricidad en climas templados [26], ya que en periodos donde se consume más electricidad, como en invierno, se presentan olas con mayor potencial energético [28].

- La energía undimotriz es mayor que $5 \mathrm{~kW} / \mathrm{m}$ al menos el 95\% del tiempo [7] y existen referencias sobre dispositivos de energía undimotriz generando electricidad sobre $90 \%$ del tiempo, en comparación al 20-30\% de los dispositivos eólicos y solares [1, 24, 29].

- Existen técnicas de construcción de plataformas marinas que son homólogas a los requerimientos de construcción de dispositivos undimotrices [25], no requiriendo, necesariamente, la investigación de nuevos métodos de construcción

- Entregan seguridad al suministro energético a zonas de aislamiento geográfico $[25,30]$.

- Amortigua el oleaje reduciendo su intensidad [1, 24-26, 31], lo que permitiría la transformación de regiones litorales en playas aptas para el baño, así como también en zonas portuarias y erosionables.

- Permite el uso alternativo de la energía obtenida, como procesos de producción de hidrógeno, potabilización del agua, entre otros [25].

\section{Desafíos}

Para conseguir los beneficios antes mencionados, hay un número de desafíos técnicos que necesitan ser resueltos para incrementar el rendimiento y desde ahí lograr equipos undimotrices competitivos en el mercado global.

El gran desafío es la conversión de movimiento oscilatorio de baja $(\sim 0.1 \mathrm{~Hz})$, aleatoria y alta intensidad en un movimiento útil para impulsar generadores con potencia de salida de calidad aceptable para la red pública. Como las olas varían en altura y periodo, su respectiva potencia varía de forma similar. Si bien la potencia media bruta puede ser predicha de antemano, esta variable de entrada tiene que ser convertida en una potencia de salida uniforme, usualmente requiriendo sistemas de almacenamiento de energía, u otros medios que permita compensar variabilidad del flujo de potencia de salida [24].

Adicionalmente, en localizaciones mar adentro la dirección del oleaje es altamente variable, por ende, los dispositivos undimotrices tienen que alinearse con amarres compatibles con las condiciones marinas existentes o ser simétricos con el fin de capturar la energía de la ola. La dirección de las olas cercanas a la costa puede ser determinada por adelantado, en gran medida, mediante el cálculo de fenómenos físicos de refracción y reflexión [24].

El desafío de capturar eficientemente este movimiento irregular tiene un impacto en el diseño del equipo. Para operar eficientemente, el dispositivo y sus correspondientes sistemas deben ser evaluados para los niveles de potencia de mayor ocurrencia. Alrededor de las Islas Británicas y el oeste de Europa, los niveles de potencia media están entre 30-70 kW/m lineal de ola [8]. Sin embargo, el equipo debe soportar las condiciones extremas de oleaje, que ocurren con poca frecuencia, pero que pueden tener niveles de potencias de más de $2000 \mathrm{~kW} / \mathrm{m}$. No solo plantea difíciles desafíos de ingeniería sino que también desafíos económicos, ya que la potencia de salida, y en consecuencia los ingresos, son obtenidos por la producción de energía de las olas que son más frecuentes, mientras que el capital requerido para la construcción del dispositivo debe cumplir con los requerimientos para soportar las olas extremas que contienen mayor energía, aun cuando estas son menos comunes [32]. También hay desafíos de diseño para mitigar el ambiente altamente corrosivo de la superficie del agua donde operan los dispositivos [26].

En el desarrollo de tecnología para la recolección de energía undimotriz, son muchas las barreras económicas y técnicas que impiden la formación de granjas de olas [33-34], siendo un punto importante la identificación de localidades óptimas. Debido a que los costos de inversión requeridos para la puesta en marcha son elevados, es útil disponer de estimaciones de potencia de salida [35]\{Reikard, 2015, Simulating and forecasting ocean wave energy in western Canada\}, que considere localidades en donde se emplazará y características morfológicas de la costa y del suelo marino, ya que dependiendo 
de éstas la distribución energética se presenta de manera particular [31]. Además, otros focos que no han sido abordados son la incertidumbre sobre el impacto generado por las granjas de olas en el mar y la costa [36-46] o el hecho de que se considere poco rentable [32].

Estudios previos han usado flujos de energía undimotriz para estimar la energía potencial. Actualmente se encuentran disponibles las matrices de varios tipos de Convertidores de Energía Undimotriz (WEC), haciendo posible la estimación de la potencia de salida [35].

Finalmente, las pesquisas desarrolladas en el área han tenido múltiples caminos. Hasta la fecha, el mundo académico ha estudiado las condiciones del mar y los métodos de conservación para impedir el deterioro de materiales, así como el diseño e interfaz conceptual de la ola primaria. Sin embargo, los métodos de uso del movimiento de la interfaz primaria son diversos. Por ello, es necesaria una evaluación detallada del sistema en totalidad ya optimizado, desarrollando sistemas aún más resistentes [24].

En este sentido, la importancia de la evaluación económica difícilmente puede ser sobreestimada, la viabilidad económica es condición sine qua non para el desarrollo de esta innovadora energía renovable; esto involucra la evaluación detallada de costos y beneficios asociados a la inversión en estas tecnologías. De esta manera, la gran mayoría de estudios sobre este campo se basan en estudios de rentabilidad [47-53], pudiendo encontrar estudios característicos para lugares en específico. Por ejemplo los realizados en Escocia [48], Reino Unido [54] e Irlanda [51-52].

Estableciendo estas tecnologías en una etapa de desarrollo e investigación inicial [47, 55-61], hace difícil estimar los costos y el rendimiento de los dispositivos e instalaciones. Además, la mayor parte de los estudios económicos actuales están sobre simplificados, y esto podría crear inseguridad en los inversionistas [62].

\section{Convertidores de energía undimotriz}

Hay un gran número de conceptos de conversión de energía undimotriz; más de 1000 técnicas de conversión de energía undimotriz han sido patentadas en Japón, Norte América, y Europa [26]. Pese a esta gran variedad de diseño, los WECs son generalmente categorizados por localización y tipo.

\section{Localización de dispositivos}

Los dispositivos se clasifican según su localización, indicados en la Figura 2, presentando características particulares para cada ubicación.

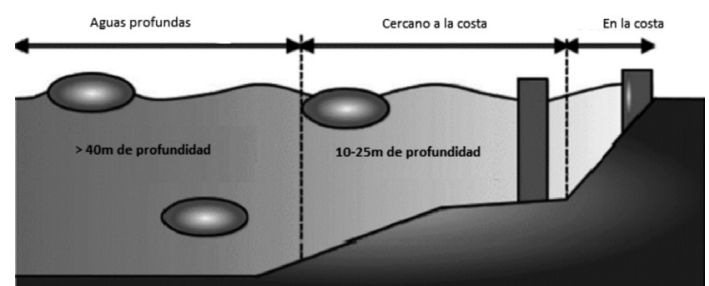

Figura 2. Esquema de localización de dispositivos según distancia de la costa [63].

\section{Los dispositivos de línea de costa (Onshore Devices)}

Estos dispositivos tienen la ventaja de estar más cerca del sistema interconectado, son fáciles de mantener, y como las olas son atenuadas a medida que viajan por aguas poco profundas tienen una menor probabilidad de condiciones extremas, lo cual reduce el riesgo de daño a los WECs. Esto conduce a una desventaja de los dispositivos situados en la costa, ya que el agua poco profunda genera potenciales undimotrices más bajos (esto puede ser parcialmente compensado por puntos de energía naturalmente concentrada). Además por la naturaleza de su localización, generalmente hay requerimientos específicos, incluyendo la geometría y geología de la costa junto con la preservación del paisaje costero, entonces los dispositivos no pueden ser diseñados para fabricación en masa [24, 63].

\section{Los dispositivos de costa (Nearshore Devices)}

Estos dispositivos están definidos como equipos que están en aguas relativamente poco profundas (no existe consenso sobre la definición de aguas poco profundas, pero se sugiere que podría ser una profundidad menor a un cuarto de la longitud de la ola [64]). Dispositivos en esta localización son a menudo fijados al fondo marino, lo que da una base fija adecuada contra la que un cuerpo oscilante puede trabajar. Como en los dispositivos de costa, en aguas poco profundas presentan una desventaja, 
generan aguas con potencia reducida, limitando la potencial recolección [24, 63].

\section{Los dispositivos mar adentro (Offshore Devices)}

Los dispositivos mar adentro están generalmente en aguas profundas aunque hay poco acuerdo sobre lo que constituye mar adentro. Decenas de metros es una definición [65], con más de 40m [64], y una profundidad superior a un tercio de la longitud de la ola [66], entre otras. El beneficio de situar un WEC en altamar es que éste puede recolectar una gran cantidad de energía por el gran contenido energético de las olas en aguas profundas [64]. No obstante, estos dispositivos son más difíciles de construir y mantener, y debido a la mayor altura y energía contenida en las olas, deben ser diseñados para sobrevivir a las condiciones más extremas, incrementando los costos de construcción. Pese a ello, se argumenta que con olas con mayor potencial, los dispositivos flotantes en aguas profundas ofrecen una mayor economía estructural [67].

Es útil señalar que la energía de la ola se produce en los movimientos de agua cerca de la superficie del mar. Cerca del $95 \%$ de la energía en una ola está localizada entre la superficie y un cuarto de la longitud de la ola por debajo de ella [64]. Además, las granjas de olas en aguas profundas son el tipo más cercano al desarrollo comercial $[63,68]$.

\section{Tipos de dispositivos convertidores}

Pese a la amplia variedad de diseño y conceptos, los WECs pueden ser clasificados en tres tipos predominantes [1, 24]:

\section{Atenuador}

Los atenuadores, como se ve en la Figura 3, se encuentran paralelos a la dirección predominante del oleaje y monta las olas. Un ejemplo de un WEC Atenuador es el Pelamis, desarrollado por Ocean Power Delivery Ltd.

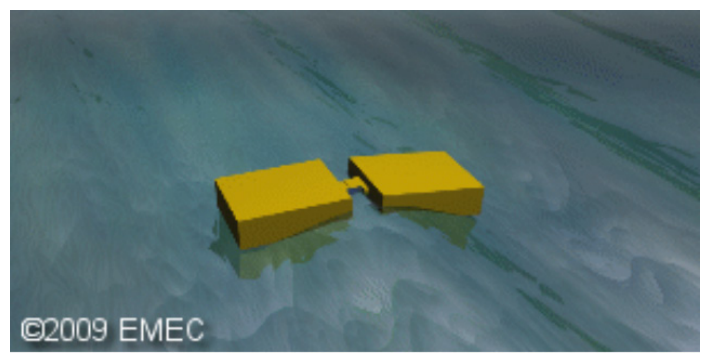

Figura 3. Dispositivo atenuador [22].

\section{Absorbedor puntual}

Un absorbedor puntual es un dispositivo que posee dimensiones pequeñas en relación con la longitud de la ola incidente. Pueden ser estructuras flotantes, como se observa en la Figura 4, que oscilan hacia arriba y hacia abajo en la superficie del agua o sumergidos debajo de ésta dependiendo del diferencial de presión. Debido a su pequeño tamaño la dirección de la onda no es importante para estos dispositivos.

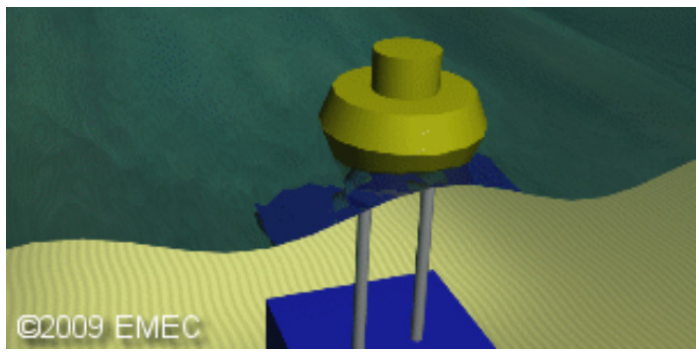

Figura 4. Dispositivo Absorbedor [22].

\section{Terminador}

Los dispositivos terminadores, generalmente tienen un eje principal paralelo al frente de olas (perpendicular a la dirección de la ola predominante) e intercepta físicamente las olas. Un ejemplo de WEC de tipo Terminador es Salter's Duck, como se muestra en la Figura 5, desarrollado en la Universidad de Edimburgo.

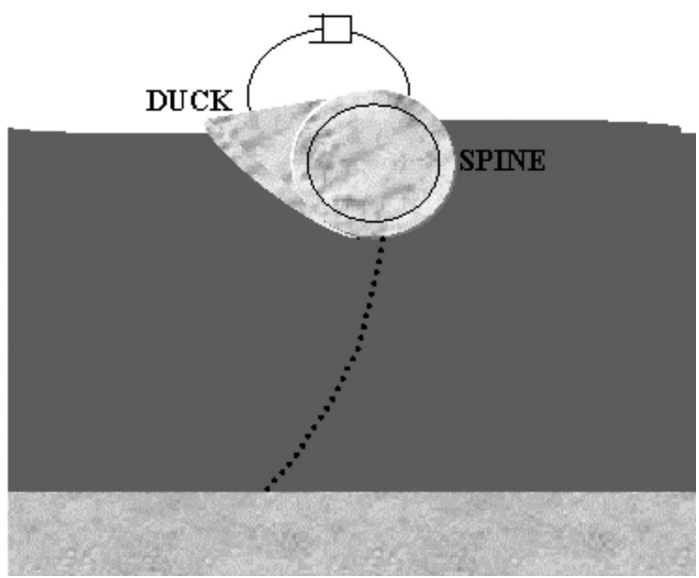

Figura 5. Dispositivo terminador [69].

\section{Modos de operación}

Dentro de las categorías anteriores hay un nivel adicional de clasificación de los dispositivos, 
determinada por su modo de funcionamiento. Algunos ejemplos significativos se dan a continuación $[1,24]$ :

\section{Sumergido por diferencial de presión (SPD)}

El dispositivo SPD, indicado en la Figura 6, es un absorbedor puntual sumergido que usa el diferencial de presión por encima del dispositivo entre la cresta y base/seno de la ola. Está compuesto por dos partes principales: una cámara cilíndrica llena de aire fijada al fondo marino con un pistón en la parte superior. Cuando la cresta de la ola pasa por encima del dispositivo, la presión de agua sobre éste comprime el aire dentro del cilindro, moviendo el pistón hacia abajo. Cuando el seno de la ola pasa por encima del equipo, la presión del agua sobre este disminuye y el cilindro superior se eleva. Una ventaja de este dispositivo es que ya que se encuentra totalmente sumergido, no está expuesto a las fuertes olas que azotan a los dispositivos flotantes [70], y reduce el impacto visual de los dispositivos. De todas formas, el mantenimiento de los equipos es un problema probable. Debido a que una parte del dispositivo se encuentra unido al fondo del mar, estos dispositivos se encuentran normalmente cerca de la costa. Un ejemplo de este dispositivo es Archimedes Wave Swing.

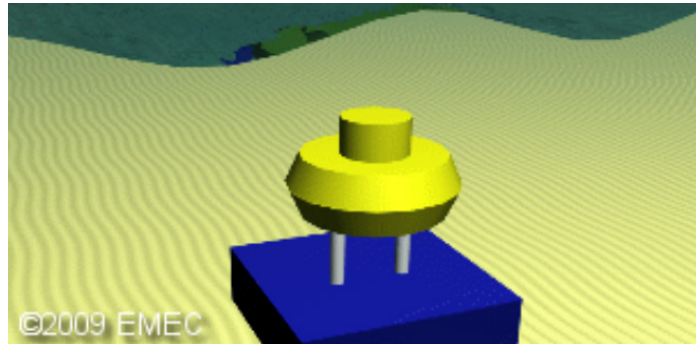

Figura 6. Dispositivo SPD [22].

\section{Convertidor de ola oscilante (OWSC)}

Un OWSC esta generalmente compuesto de un deflector de bisagras, como se ve en la Figura 7, posicionado perpendicular a la dirección de las olas (un terminador), que se mueve hacia adelante y hacia atrás aprovechando la velocidad horizontal de la ola.

\section{Columna de agua oscilante (OWC)}

Un OWC consta de una cámara con dos aperturas. La apertura inferior, ubicada bajo el nivel del mar permite el ingreso de la masa de agua desplazadas

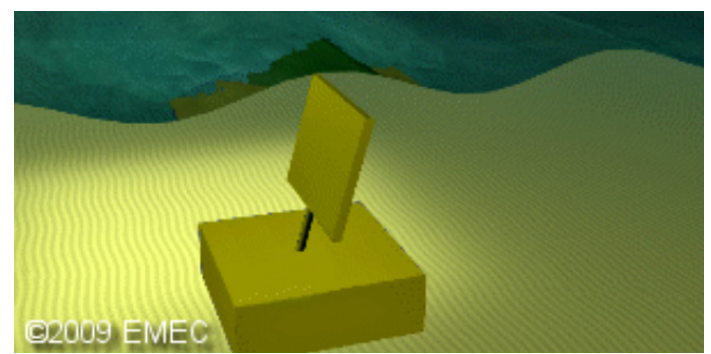

Figura 7. Dispositivo OWSC [22].

por la fuerza de las olas a la cámara, movilizando la masa de aire hacia la apertura superior y expulsando el aire hacia la atmosfera a través de una turbina. Como el agua es retirada, el aire es aspirado luego a la cámara.

La turbina Wells de baja presión es utilizada a menudo ya que rotan en la misma dirección independiente de la dirección del flujo, quitando la necesidad de rectificar el flujo de aire. Se ha sugerido que la ventaja del OWC es la simplicidad y solidez.

Hay ejemplos de OWCs como absorbedores puntuales, como también están siendo construidos en la costa, donde actúan como terminadores, como se muestra en la Figura 8.

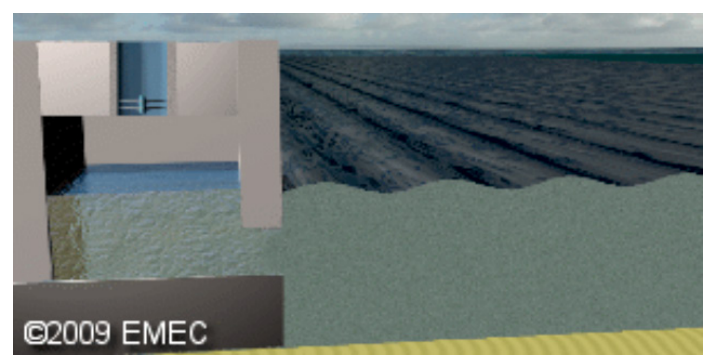

Figura 8. Dispositivo OWC [22]

El concepto de OWC ha sido propuesto por Oceanlinx, un desarrollador undimotriz australiano, en un dispositivo anclado cerca de la costa.

\section{Dispositivo de desbordamiento/Inundable (OD)}

Un OD captura el agua de las olas incidentes en un depósito sobre el nivel del mar, y luego descarga el agua hacia el mar por medio de turbinas, expuesto en la Figura 9. Un ejemplo de este es el Wave Dragon [71]. 


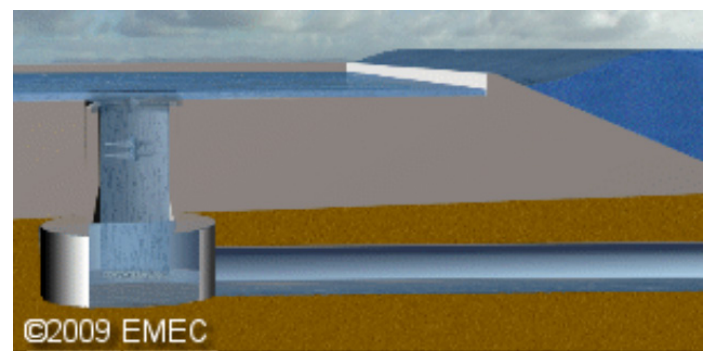

Figura 9. Dispositivo de desbordamiento [22].

Este dispositivo utiliza reflectores curvos para recolectar las olas en su centro, las olas fluyen por una rampa e ingresan por la parte superior a un depósito elevado, donde el agua es regresada al mar a través de un número de turbinas low-head.

\section{RESULTADOS/DISCUSIÓN}

Las reservas de recursos energéticos no renovables han disminuido de forma creciente, dejando a países como Chile, principalmente importador de recursos energéticos, en situación de riesgo potencial [2].

Estudios desarrollados [6, 72] demuestran que Chile posee un considerable potencial energético undimotriz, estableciendo a éste como el recurso renovable más importante del país [72], aun cuando este no es aprovechado en la actualidad. Se cuenta con más de $4500 \mathrm{~km}$ de costa, haciendo del país uno de los lugares más propicios del mundo para la generación de este tipo de energía [72].

Debido a la extensión de la costa, se estima que los niveles de energía aumentan de forma exponencial de norte a sur, encontrándose dentro del intervalo $25-110 \mathrm{~kW} / \mathrm{m}$. De igual manera, presenta un incremento en el potencial mientras se aleja de la costa [7].

Según un estudio realizado en el sitio $21,04^{\circ} \mathrm{S}$ $70,84^{\circ} \mathrm{O}$, situado en aguas profundas frente a la región de Tarapacá, presenta un porcentaje cercano al $87 \%$ de olas que superan los 2 metros, que dan como resultado potencias superiores a los $25 \mathrm{~kW} / \mathrm{m}$, $\mathrm{y}$ con un factor de estacionalidad que hace que este pueda incrementar hasta los $61,94 \mathrm{~kW} / \mathrm{m}$, como indica la Tabla 1 [28]. A esto se debe considerar un factor base, que el recurso mínimo de energía undimotriz para generar electricidad es de $5 \mathrm{~kW} / \mathrm{m}$ [73].
Además. la región de Tarapacá cuenta con un conjunto de fortalezas y ventajas comparativas, no vinculadas a la energía, respecto a otras regiones del país y lugares del mundo, que deben ser utilizados como catalizadores de la economía regional, tales como [74]:

- Situación geográfica y logística privilegiada.

- Existencia de recursos naturales valorados en el mercado internacional.

- Potencialidad turística.

Según la información mostrada en la Tabla 2, el Sistema Interconectado del Norte Grande (SING), para el periodo indicado, solo $4 \%$ de la energía inyectada fue ERNC [71], situación que dista del objetivo inicial indicado en la agenda energética de alcanzar 20\% de ERNC para el año 2020 [75], y aun más lejano del $70 \%$ plasmado en la Hoja de Ruta 2050 [3]; marcando como antecedente que las principales fuentes renovables son solar y eólica.

Tabla 1. Valores medios de la altura significativa, periodo medio, potencia y dirección de propagación de olas [28].

\begin{tabular}{|l|c|c|c|}
\hline Mes & $\begin{array}{c}\text { Altura } \\
\text { Significativa }\end{array}$ & $\begin{array}{c}\text { Período } \\
\text { medio }\end{array}$ & Potencia \\
\hline & $(\mathrm{m})$ & $(\mathrm{s})$ & $(\mathrm{kM} / \mathrm{m})$ \\
\hline Ene & 2,28 & 10,83 & 30,88 \\
\hline Feb & 2,17 & 10,95 & 26,59 \\
\hline Mar & 2,28 & 11,45 & 32,55 \\
\hline Abr & 2,67 & 11,70 & 43,12 \\
\hline May & 2,72 & 12,02 & 47,75 \\
\hline Jun & 2,99 & 11,53 & 56,45 \\
\hline Jul & 3,14 & 11,69 & 61,91 \\
\hline Ago & 2,98 & 12,03 & 59,11 \\
\hline Sep & 2,64 & 10,80 & 39,26 \\
\hline Oct & 2,90 & 11,78 & 53,46 \\
\hline Nov & 2,43 & 11,00 & 33,41 \\
\hline Dic & 2,31 & 10,68 & 30,64 \\
\hline
\end{tabular}

Además, como muestra la Tabla 3, el 88,80\% de la venta de energía del SING ha sido a clientes libres [75], quienes son afectados por los altos precios debido la producción de electricidad basada en diesel, lo que establece un costo marginal más alto a 85,4 USD/MWh a junio de 2016, según la consultora del sector energético SYSTEP [76]. 
La región de Tarapacá ha presentado un crecimiento económico considerable en las últimas dos décadas, principalmente debido a la Industria minera y Comercio, nacional e internacional, por medio de la Zona Franca de Iquique, los que conforman un motor económico para la región [74], que para el 2014 componían el 79,8\% del PIB regional [77].

Considerando el contexto económico de Chile, y la condición minera tácita del Norte Grande, es fácil comprender que los costos de la energía para la industria minera incrementan los costos de explotación del cobre [74], debiendo comprender que para el año 2013 la minería del cobre alcanzo un consumo de $22057 \mathrm{GWh}$, equivalente al 32\% del consumo energético a nivel país [78].

Tabla 2. Generación bruta anual por tipo de combustible al 07-09, 2016 [75].

[\%] Generación Bruta Anual por tipo Combustible desde el 01-01-2016 al 07-09-2016

\begin{tabular}{|l|c|}
\hline \multicolumn{1}{|c|}{ Tipo Combustible } & \% \\
\hline Carbón & 58,3 \\
\hline Cogeneración & $0,5 \%$ \\
\hline Diesel & $4,8 \%$ \\
\hline Eólico & 0,9 \\
\hline Fuel Oil ${ }^{\circ}$ 6 & 0,4 \\
\hline Gas Natural & 7,6 \\
\hline Hidro & 0,3 \\
\hline Solar & 2,8 \\
\hline Total Anual & $75,6 \%$ \\
\hline
\end{tabular}

Tabla 3. Ventas a clientes libres y regulados, 01/01 a 07/09, 2016.

\begin{tabular}{|l|c|c|}
\hline \multicolumn{3}{|c|}{ Ventas de Clientes Libres y Regulados } \\
\hline Enero a Julio 2016 & [GWh] & {$[\%]$} \\
\hline Regulado & 1121,35 & 11,20 \\
\hline Libre & 8891,49 & 88,80 \\
\hline
\end{tabular}

La posibilidad de suplir el 4,8\% de generación eléctrica vía Diesel, podría generar una disminución aun mayor al precio de la electricidad respecto a la obtenida hasta ahora [75].

Las estrategias del desarrollo regional de la región de Tarapacá se basan en un equilibrio en lo social, medioambiental y previendo el uso y gestión de recursos naturales [79].

El desarrollo y potenciamiento de sectores privilegiados se establece para generar una economía fortalecida. Sin embargo, se plantea el objetivo de potenciar economías sustitutivas, como ERNC, acuicultura y agricultura del desierto. En particular, la región de Tarapacá posee ventajas, contando con alrededor de $400 \mathrm{~km}$ de agua en su extenso litoral, y reúne variables, como temperatura, nutrientes, entre otras, que hacen aptas sus costas para el desarrollo del sector acuícola, o el cultivo de especies no tradicionales de la zona como cultivos cítricos, subtropicales y tropicales, que han aumentado significativamente, pero que se encuentran limitados por los recursos hídricos [74].

El elemento que causa más preocupación es que de la capacidad instalada del SING [80] solo el 4\% corresponde a ERNC, como se expone en la Figura 10, equivalente a $207 \mathrm{MW}$ en capacidad instalada, insignificante frente a los 4.142,8 MW de capacidad total [81] y si bien existen proyectos en construcción, que a nivel nacional proporcionarán $2.378 \mathrm{MW}$ de capacidad instalada en fuentes renovables [82], aún no se está tomando en consideración las energías marinas como una fuente confiable de producción energética.

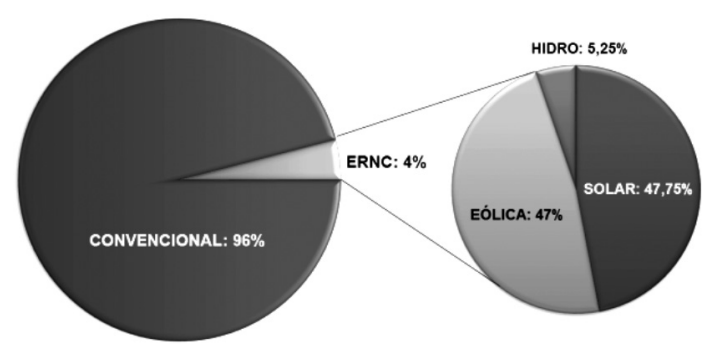

Figura 10. Distribución porcentual de capacidad instalada por combustible, 2015 [80].

Una característica clave a favor que se presenta, tiene relación con los stocks de seguridad. Las generadoras, como toda industria, deben contar con stocks de seguridad y también se exponen al riesgo de cortes de suministros de combustibles convencionales [62]. El año 2002, Argentina cortó el suministro de gas a Chile lo que provocó un incremento de casi el 300\% de las tarifas, ralentizando el crecimiento económico 
e incrementando las emisiones de carbono por la utilización de combustibles como el diesel [83].

El recurso básico para la energía undimotriz son las olas, un recurso libre y que solo se ve afectado por el efecto del viento, es por ello que generar una planificación efectiva y tener sistemas de almacenamiento de electricidad para generar una alimentación continua a la red permite contar con una fuente de energía más estables en términos de generación, y en consecuencia, evitar grandes pérdidas económicas ocasionadas por problemas de faltantes.

Las empresas de la región no se sitúan en zonas costeras por motivos logísticos, al no contar con agua y electricidad. Principalmente debido a la normativa y reglamentación vigente, que exige la disponibilidad de agua destinada al consumo humano y necesidades básicas de higiene y aseo personal [84].

Además, los problemas de abastecimiento de agua, en el Norte Grande, interfieren procesos económicos como la agricultura, minería y actividad industrial en general. Líneas estratégicas en el país se enfocan en la investigación de nuevas fuentes de abastecimiento de agua, como las plantas desalinizadoras, lo cual influye que gran parte del litoral costero no es utilizado por el sector industrial, simplemente por no cumplir con el reglamento sanitario de contar con agua potable [79].

Esta necesidad puede ser satisfecha a través de la energía undimotriz, ya que podría proveer de electricidad a plantas desalinizadoras, teniendo presente que las olas de la región de Tarapacá cuenta con la potencia suficiente para el funcionamiento de estas unidades. Dado que en procesos de electrodiálisis u osmosis inversa el consumo energético es cercano a $1 \mathrm{kWh} / \mathrm{m}^{3}$ [85].

Finalmente, se debe considerar el factor del desarrollo económico, la puesta en marcha de una nueva central eléctrica, implica la generación de nuevos puestos de trabajo, y en particular considerando esta nueva fuente de energía, ya que además se debe pensar en el clúster local que se desarrolla, el aprendizaje de técnicas y conocimientos necesario para desempeñarse en determinada industria. Las grandes empresas mineras de la región de Tarapacá, no solo han contribuido al PIB nacional y regional, sino que han instaurado un ecosistema propicio para la creación de nuevas empresas, personal calificado con líneas de responsabilidad social empresarial, y el desarrollo nuevas industrias.

\section{CONCLUSIONES}

Al ver la variabilidad mensual del potencial undimotriz, se reconoce un comportamiento estacional, incrementando su potencial en invierno, validando de esta manera la tendencia similar a la demanda energética, que también incrementa en dicha estación.

Se plantea la implementación de granjas de olas en las costas de la región de Tarapacá, ya que si bien presentan un potencial reducido en comparación con el resto de Chile y no está situada dentro de las latitudes optimas, el contar con más de $20 \mathrm{~kW} / \mathrm{m}$ de longitud de ola, está muy por sobre de la media mundial, sin considerar que se plantea que una potencia sobre $5 \mathrm{~kW} / \mathrm{m}$ ya es suficiente para tener una generación eléctrica con resultados aceptables, lo que hace apta a la región para recolectar el recurso undimotriz, todo sujeto a estudios de factibilidad técnica-económica que sustente su construcción e implementación.

En relación a los dispositivos WECs, sin perjuicio de las consideraciones del suelo marino en la región de Tarapacá, se entiende que cualquier tipo de dispositivos haría posible la generación de electricidad vía energía undimotriz. No obstante, se debe poner énfasis en los dispositivos de tipo offshore, en particular los absorbedores puntuales, ya que son dispositivos menos invasivos, incluso sumergibles, presentan alto grado de recuperación y se ubican en zonas de fuerte oleaje que entregan altos potenciales undimotrices.

La implementación de energía undimotriz, debiese considerarse como un factor que favorecerá el encadenamiento productivo regional, ya que podría proveer de electricidad y agua potable a zonas no conectadas a las redes de servicios públicos, proporcionando la oportunidad de desarrollar emprendimientos en zonas donde hasta el momento no era posible. Además, pudiendo ser el eje de los sectores económicos locales. 
En referencia a los costos de la implementación y desarrollo de energía undimotriz, se sabe que los costos de inversión son altos. Sin embargo, se debe tener en consideración que a diferencia de las fuentes convencionales de electricidad, no es necesaria la compra del recurso energético primario, y en consecuencia debe entenderse que la energía en si es mucho más económica que la fuentes actuales.

Chile, a lo largo de su costa, cuenta con alrededor del $74 \%$ del potencial de la costa oeste de Sudamérica en recurso undimotriz, es por esto que se debe considerar la oportunidad que esto trae consigo. Es claro que existe una oportunidad para Chile de jugar un papel relevante en la Investigación y desarrollo de tecnología de recuperación undimotriz, al establecer una capacidad de fabricación que no se presenta en industrias más consolidadas, como la energía eólica y solar, donde el equipamiento es principalmente importado.

Aunque existen incertidumbres en relación al costo de producir electricidad a partir de la energía undimotriz, el potencial de reducción de costos es alto al entrar en etapa de maduración la tecnología undimotriz, ya que este suceso no solo ocurre en este tipo de tecnologías sino que es transversal en las actividades de investigación y desarrollo de diversas industrias.

El desarrollo de la energía undimotriz puede determinar el encadenamiento de los seis sectores estratégicos, siendo el eje de desarrollo productivo de la región de Tarapacá, desarrollando tecnología recuperadora de energía potenciando el sector ERNC, proveer de energía al sector minero, posibilitar el desarrollo de la agricultura del desierto suministrando agua potable, compatibilizando dispositivos con la industria acuícola, y potenciando el comercio y turismo estableciendo a la región de Tarapacá como un foco de desarrollo científico y tecnológico.

Aunque la energía undimotriz, se presenta como una opción atractiva, no existe una profundización actual de la implementación y desarrollo de esta en la región de Tarapacá, lo que impide los procesos de toma de decisiones sobre la implementación de estas tecnologías, abriendo un campo de investigación en esta área.

\section{REFERENCIAS}

[1] I. Lopez, J. Andreu, S. Ceballos, I.M. de Alegria and I. Kortabarria. "Review of wave energy technologies and the necessary powerequipment". Renewable \& Sustainable Energy Reviews. Vol. 27, pp. 413-434. November, 2013.

[2] Ministerio de Energía. "Estrategia Nacional de Energía 2012-2030”. 2012. URL: http:// www.energia.gob.cl/

[3] Gobierno de Chile. 2015. "Hoja de ruta 2050". URL: http://www.energia.gob.cl/sites/default/ files/hoja_de_ruta_cc_e2050.pdf

[4] D.J. Arent, A. Wise and R. Gelman. "The status and prospects of renewable energy for combating global warming". Energy Economics. Vol. 33 No 4, pp. 584-593. July, 2011.

[5] H. Jeffrey, B. Jay and M. Winskel. "Accelerating the development of marine energy: Exploring the prospects, benefits and challenges". Technological Forecasting and Social Change. Vol. $80 \mathrm{~N}^{\circ}$ 7, pp. 13061316. September, 2013.

[6] J. Cruz, M.D. Thomson, E. Stavroulia, and R.I. Rawlinson-Smith. "Preliminary Site Selection-Chilean Marine Energy Resources". Garrad Hassan. 2009.

[7] "Energía marina en Chile. Avanzando en el desarrollo del recurso chileno". Errazuriz \& Asociados. British Embassy Santiago. The University of Edinburgh. 2013.

[8] H. Polinder and M. Scuotto. "Wave energy converters and their impact on power systems". 2005 International Conference on Future Power Systems, pp. 9. 2005.

[9] S.H. Salter. "Wave power" Nature. Vol. 249 $\mathrm{N}^{\circ}$ 5459, pp. 720-724. 1974.

[10] A. Akpinar and M.I. Komurcu. "Assessment of wave energy resource of the Black Sea based on 15-year numerical hindcast data". Applied Energy. Vol. 101, pp. 502-512. January, 2013.

[11] H. Bernhoff, E. Sjostedt and M. Leijon. "Wave energy resources in sheltered sea areas: A case study of the Baltic Sea". Renewable Energy. Vol. $31 \mathrm{~N}^{\circ}$ 13, pp. 2164-2170. October, 2006.

[12] Z. Defne, K.A. Haas and H.M. Fritz. "Wave power potential along the Atlantic coast of the southeastern USA". Renewable Energy. Vol. $34 \mathrm{~N}^{\mathrm{o}} 10$, pp. 2197-2205. October, 2009.

[13] G. Iglesias and R. Carballo. "Wave energy potential along the Death Coast (Spain)". 
Energy. Vol. $34 \mathrm{~N}^{\circ}$ 11, pp. 1963-1975. November, 2009.

[14] G. Iglesias, M. Lopez, R. Carballo, A. Castro, J. A. Fraguela and P. Frigaard. "Wave energy potential in Galicia (NW Spain)". Renewable Energy. Vol. $34 \mathrm{~N}^{\mathrm{o}}$ 11, pp. 2323-2333. November, 2009.

[15] G. Iglesias and R. Carballo. "Offshore and inshore wave energy assessment: Asturias (N Spain)". Energy. Vol. 35 No 5, pp. 19641972. May, 2010.

[16] G. Iglesias and R. Carballo. "Wave energy and nearshore hot spots: The case of the SE Bay of Biscay". Renewable Energy. Vol. 35 $\mathrm{N}^{\mathrm{o}}$ 11, pp. 2490-2500. November, 2010.

[17] G. Iglesias and R. Carballo. "Wave energy resource in the Estaca de Bares area (Spain)". Renewable Energy. Vol. 35 N $^{\circ} 7$, pp. 15741584. July, 2010.

[18] G. Iglesias and R. Carballo. "Wave power for La Isla Bonita”. Energy. Vol. 35 Nº 12, pp. 5013-5021. December, 2010.

[19] G. Iglesias and R. Carballo. "Choosing the site for the first wave farm in a region: A case study in the Galician Southwest". Energy. Vol. 36 $\mathrm{N}^{\circ}$ 9, pp. 5525-5531. September, 2011.

[20] G. Iglesias and R. Carballo. "Wave resource in El Hierro-an island towards energy selfsufficiency". Renewable Energy. Vol. 36 $\mathrm{N}^{\mathrm{o}}$ 2, pp. 689-698. February, 2011.

[21] G. Mork, S. Barstow, A. Kabuth and M. T. Pontes. "Assessing the global wave energy potential". The American Society of Mechanical Engineers, pp. 447-454. 2010.

[22] M. Moschini F. "Acelerando el desarrollo de la energía marina para Chile". Fundación Chile. 2015. URL: http://ift-energy.cl/ wp-content/uploads/ 2015/04/1-FundacionChile-Moschini.pdf

[23] J.L. Villate. "Situación actual de las energías marinas y perspectivas de futuro". Seminario anual de Automática, Electrónica e Instrumentación (SAAEI). 2010.

[24] B. Drew, A.R. Plummer and M.N. Sahinkaya. "A review of wave energy converter technology". Proceedings of the Institution of Mechanical Engineers Part a-Journal of Power and Energy. Vol. 223 N A8, pp. 887902. December, 2009.

[25] J.F. Chozas. "Energía Undimotriz: Una aproximación al aprovechamiento de la energía de las olas para la generación de electricidad". Lambert Academic Publishing. 2012.

[26] A. Clement, P. McCullen, A. Falcão, A. Fiorentino, F. Gardner, K. Hammarlund, G. Lemonis, T. Lewis, K. Nielsen, S. Petroncini, M. Pontes, P. Schild, BengtOlov Sjöström, H. Sørensen and T. Thorpe. "Wave energy in Europe: current status and perspectives". Renewable \& Sustainable Energy Reviews. Vol. 6 N $^{\circ}$ 5, pp. 405-431. Pii s1364-0321(02)00009-6. October, 2002.

[27] T.W. Thorpe. "A brief review of wave energy". Harwell Laboratory. Energy Technology Support Unit London. United Kingdom. 1999.

[28] "Informe de Evaluación del Recurso Marino". Ministerio de Energía, Gobierno de Chile. Facultad de Ciencias Físicas y Matemáticas. Universidad de Chile. 4 de diciembre de 2015.

[29] R. Pelc and R. M. Fujita. "Renewable energy from the ocean". Marine Policy. Vol. $26 \mathrm{~N}^{\circ} 6$, pp. 471-479. Pii s0308-597x(02)00045-3. November, 2002.

[30] I. Martínez-Neghme. "Un sistema de energía undimotriz para zonas aisladas". Revismar. Vol. 3, pp. 274-279. 2014.

[31] C.J. Daly, K.R. Bryan and C. Winter. "Wave energy distribution and morphological development in and around the shadow zone of an embayed beach". Coastal Engineering. Vol. 93, pp. 40-54. 2014.

[32] M. Leijon, O. Danielsson, M. Eriksson, K. Thorburn, H. Bernhoff, J. Isberg, J. Sundberg, I. Ivanova, E. Sjöstedt, O. Ägren, K.E. Karlsson and A. Wolfbrandt. "An electrical approach to wave energy conversion". Renewable Energy. Vol. 31 N $^{\circ}$ 9, pp. 13091319. July, 2006.

[33] M. Esteban and D. Leary. "Current developments and future prospects of offshore wind and ocean energy". Applied Energy. Vol. $90 \mathrm{~N}^{\mathrm{o}}$ 1, pp. 128-136. 2012.

[34] R.A. Arinaga and K.F. Cheung. "Atlas of global wave energy from 10 years of reanalysis and hindcast data". Renewable Energy. Vol. 39 NN $^{\circ}$, pp. 49-64. 2012.

[35] G. Reikard, B. Robertson, B. Buckham, J.R. Bidlot and C. Hiles. "Simulating and forecasting ocean wave energy in western 
Canada". Ocean Engineering. Vol. 103, pp. 223-236. July, 2015.

[36] J. Abanades, D. Greaves and G. Iglesias, "Wave farm impact on the beach profile: A case study". Coastal Engineering. Vol. 86, pp. 36-44. April, 2014.

[37] R. Carballo and G. Iglesias. "Wave farm impact based on realistic wave-WEC interaction”. Energy. Vol. 51, pp. 216-229. 2013.

[38] C. Frid, E. Andonegi, J. Depestele and E. Kenchington. "The environmental interactions of tidal and wave energy generation devices". Environmental Impact Assessment. Vol. 32 $\mathrm{N}^{\circ}$ 1, pp. 133-139. 2012.

[39] M. Kadiri, R. Ahmadian, B. BockelmannEvans, W. Rauen and R. Falconer. "A review of the potential water quality impacts of tidal renewable energy systems". Renewable \& amp; Sustainable Energy Review. Vol. 16 $\mathrm{N}^{\circ}$ 1, pp. 329-341. January, 2012.

[40] L. Margheritini, A.M. Hansen and P. Frigaard. "A method for EIA scoping of wave energy converters-based on classification of the used technology". Environmental Impact Assessment Review. Vol. $32 \mathrm{~N}^{\circ}$ 1, pp. 33-44. January, 2012.

[41] D.L. Millar, H.C.M. Smith and D.E. Reeve. "Modelling analysis of the sensitivity of shoreline change to a wave farm". Ocean Engineering. Vol. $34 \mathrm{~N}^{\circ}$ 5-6, pp. 884-901. April, 2007.

[42] A. Palha, L. Mendes, C.J. Fortes, A. BritoMelo and A. Sarmento. "The impact of wave energy farms in the shoreline wave climate: Portuguese pilot zone case study using Pelamis energy wave devices". Renewable Energy. Vol. $35 \mathrm{~N}^{\circ}$ 1, pp. 62-77. January, 2010.

[43] S.P. Neill, E.J. Litt, S.J. Couch and A.G. Davies. "The impact of tidal stream turbines on large-scale sediment dynamics". Renewable Energy. Vol. 34 N ${ }^{\circ} 12$, pp. 28032812. December, 2009.

[44] V. Ramos, R. Carballo, M. Alvarez, M. Sanchez and G. Iglesias. "Assessment of the impacts of tidal stream energy through high-resolution numerical modeling". Energy. Vol. 61, pp. 541-554. November, 2013.

[45] D.E. Reeve, Y. Chen, S. Pan, V. Magar, D.J. Simmonds and A. Zacharioudaki. "An investigation of the impacts of climate change on wave energy generation: The Wave Hub, Cornwall, UK”. Renewable Energy. Vol. 36 No 9, pp. 2404-2413. September, 2011.

[46] M. Sanchez, R. Carballo, V. Ramos and G. Iglesias. "Tidal stream energy impact on the transient and residual flow in an estuary: A 3D analysis". Applied Energy. Vol. 116, pp. 167-177. March, 2014.

[47] Y. Li, B.J. Lence and S.M. Calisal. "An integrated model for estimating energy cost of a tidal current turbine farm". Energy Conversion and Management. Vol. $52 \mathrm{~N}^{\circ} 3$, pp. 1677-1687. March, 2011.

[48] G.J. Allan, I. Brydenb, P. McGregora, T. Stallardd, J. Swalesa, K. Turnera and R. Wallaceb. "Concurrent and legacy economic and environmental impacts from establishing a marine energy sector in Scotland". Energy Policy. Vol. $36 \mathrm{~N}^{\mathrm{o}}$ 7, pp. 2734-2753. July, 2008.

[49] G. Allan, M. Gilmartin, P. McGregor, and K. Swales. "Levelised costs of Wave and Tidal energy in the UK: Cost competitiveness and the importance of \&quot;banded\&quot; Renewables Obligation Certificates". Energy Policy. Vol. $39 \mathrm{~N}^{\circ}$ 1, pp. 23-39. January, 2011.

[50] G.J. Dalton, R. Alcorn and T. Lewis. "Case study feasibility analysis of the Pelamis wave energy convertor in Ireland, Portugal and North America". Renewable Energy. Vol. $35 \mathrm{~N}^{\circ}$ 2, pp. 443-455. February, 2010.

[51] G.J. Dalton, R. Alcorn and T. Lewis. "A 10 year installation program for wave energy in Ireland: A case study sensitivity analysis on financial returns". Renewable Energy. Vol. $40 \mathrm{~N}^{\circ}$ 1, pp. 80-89. April, 2012.

[52] J.P. Deane, G. Dalton and B.P.O. Gallachoir. "Modelling the economic impacts of 500 MW of wave power in Ireland". Energy Policy, Vol. 45, pp. 614-627. June 2012.

[53] M. O'Connor, T. Lewis and G. Dalton. "Operational expenditure costs for wave energy projects and impacts on financial returns". Renewable Energy. Vol. 50, pp. 1119-1131. February, 2013.

[54] G. Allan, M. Gilmartin, P. McGregor and K. Swales. "Levelised costs of Wave and Tidal energy in the UK: Cost competitiveness and the importance of "banded" Renewables 
Obligation Certificates". Energy Policy. Vol. $39 \mathrm{~N}^{\circ}$ 1, pp. 23-39. January, 2011.

[55] M. Borg, M. Collu and F.P. Brennan, "Use of a Wave Energy Converter as a Motion Suppression Device for Floating Wind Turbines". Energy Procedia. Vol. 35, pp. 223233. 2013.

[56] R. Carballo and G. Iglesias. "A methodology to determine the power performance of wave energy converters at a particular coastal location". Energy Conversion and Management. Vol. 61, pp. 8-18. September, 2012.

[57] I. Lopez and G. Iglesias. "Efficiency of OWC wave energy converters: A virtual laboratory". Applied Ocean Research. Vol. 44, pp. 63-70. January, 2014.

[58] H. Fernandez, G. Iglesias, R. Carballo, M. Sánchez. "The new wave energy converter WaveCat: Concept and laboratory tests". Marine Structures. Vol. 29 No 1, pp. 58-70. December, 2012.

[59] F.O. Rourke, F. Boyle and A. Reynolds, "Marine current energy devices: Current status and possible future applications in Ireland". Renewable and Sustainable Energy Reviews. Vol. 14 N $^{\circ} 3$, pp. 1026-1036. 2010.

[60] V. Ramos and G. Iglesias. "Performance assessment of Tidal Stream Turbines: A parametric approach". Energy Conversion and Management. Vol. 69, pp. 49-57. May, 2013.

[61] D. Vicinanza, P. Contestabile, J.Q.H. Norgaard and T.L. Andersen. "Innovative rubble mound breakwaters for overtopping wave energy conversion". Coastal Engineering. Vol. 88, pp. 154-170. June 2014.

[62] S. Astariz and G. Iglesias. "The economics of wave energy: A review". Renewable and Sustainable Energy. Vol. 45, pp. 397-408. 2015.

[63] A.F.d.O. Falcão. "Wave energy utilization: A review of the technologies." Renewable and Sustainable Energy. Vol. 14 № 3, pp. 899918. 2010.

[64] L.J. Duckers."Wave energy; crests and troughs". Renewable Energy. Vol. 5 № 5-8, pp. 1444-1452. 1994.

[65] J. Callaghan and R. Boud. "Future Marine Energy. Results of the Marine Energy Challenge: Cost competitiveness and growth of wave and tidal stream energy". Carbon trust. 2006.

[66] J. Falnes. "A review of wave-energy extraction". Marine Structures. Vol. $20 \mathrm{~N}^{\circ} 4$, pp. 185-201. October 2007.

[67] U. Korde. "Control system applications in wave energy conversion". In OCEANS 2000 MTS/IEEE Conference and Exhibition. Vol. 3, pp. 1817-1824: IEEE. 2000.

[68] J. Cruz. Ocean wave energy: current status and future prespectives. Springer Science \& Business Media. 2007.

[69] "WAVE the conversion of the power of ocean waves to electricity". Energy Systems Research Unit University of Strathclyde. Available: http://www.esru.strath.ac.uk/ EandE/Web_sites/98-9/offshore/wave.htm.

[70] N. Baker and M. Mueller. "Direct Drive Wave Energy Converters". Revue des Energies renouvelables. Vol. $4 \mathrm{~N}^{\circ} 2$ pp. 1-7. 2001.

[71] WaveDragonApS. "WaveDragon ...for a better future". URL: http://www.wavedragon.net/. 2005

[72] P. Monárdez, H. Acuña and D. Scott. "Evaluation of the potential of wave energy in Chile". pp. 801-809: American Society of Mechanical Engineers.

[73] T. Denniss. "Comparing the variability of wind speed and wave height data," Energetech Australia. 2005.

[74] "Estrategia regional de innovación. Región de Tarapacá". Subsecretaria de Desarrollo Regional y Administrativo. AGCI. Ministerio de Relaciones Exteriores. Gobierno Regional. Región de Tarapaca. Gobierno de Chile. Unión europea. Programa innovación y competitividad UE-Chile. 2014.

[75] "Resumen Ejecutivo de la Operación". Centro de Despacho Económico de Carga del Sistema Interconectado del Norte Grande. 2016.

[76] "Reporte mensual del Sector Eléctrico SIC y SING". Systep Ingeniería\&Diseño S.A. Vol. $9 \mathrm{~N}^{\circ}$ 8. Agosto 2016. URL: http://systep. cl/ documents/reportes/082016_Systep_ Reporte_Sector_Electrico.pdf

[77] Estadísticas de empresas por rubro y región 2005-2014. 2015. URL: http://www.sii.cl/ estadisticas/empresas_rubro.htm

[78] C. Minero. Energía y cambio climático. 2015. URL: http://www.consejo- 
minero. cl/ambitos-estrategicos/ energia-y-cambio-climatico/

[79] "Chile 2020. Obras Públicas para el Desarrollo". Ministerio de Obras Públicas. Gobierno de Chile. 2010. URL: http://www. mop.cl/Presa/ Documents/Agenda\%2020142020 pdigital.pdf

[80] "Capacidad Instalada por Combustible (MW)". Centro de Despacho Económico de Carga del Sistema Interconectado del Norte Grande. Periodo 2006-2015. 2015. URL: http://cdec2.cdec-sing.cl/portal/ page? pageid $=33,44061 \& \_$dad $=$portal\& schema $=$ PORTAL

[81] "Boletín del Mercado Eléctrico. Sector Generación". Generadoras de Chile. Noviembre, 2015. URL: http://generadoras.cl/wp-content/ uploads/Bolet $\%$ C3\%ADn-AGG-SectorGeneraci\%C3\%B3n-Noviembre-2015.pdf
[82] D. Álvarez López. "Reporte CIFES. Energías Renovables en el Mercado Eléctrico Chileno". Centro Nacional para la Innovación y Fomento de las Energías Sustentables. Agosto, 2015. URL: http:// www.cifes.gob.cl/boletin/2015/agosto/ Reporte_CIFES_Agosto15 3.pdf

[83] V. Ibarra. "Corte del gas argentino triplicó tarifas, restó crecimiento al país y ensució matriz energética". in Economía y Negocios. Enero, 2013.

[84] Decreto 594/2000. “Reglamento sobre condiciones sanitarias y ambientales basicas en los lugares de trabajo". 2000.

[85] D. Avila-Prats, R. Alesanco-García, and J. Veliz-Alonso. "Sistemas híbridos con base en las energías renovables para el suministro de energía a plantas desaladoras". Ingeniería Mecánica. Vol. 14, pp. 22-30. 2011. 